NEW RESULTS

\title{
Diversity patterns across 1,800 chloroplast genomes of wild (Oryza rufipogon Griff.) and cultivated rice (O. sativa L.)
}

\author{
Peter Civáň \& Terence A. Brown
}

Manchester Institute of Biotechnology, School of Earth and Environmental Sciences, University of Manchester, Manchester, UK.

corresponding author: Peter Civáň (peter.civan@manchester.ac.uk)

\begin{abstract}
Cultivated Asian rice (O. sativa L.) comprises several groups with distinct ecological requirements and culinary uses. While the two subspecies of $O$. sativa - indica and japonica - have been subjected to a multitude of genetic and genomic analyses, less is known about the origins and diversity of the agronomically marginal groups - aus and aromatic rice. Here we reconstructed complete chloroplast genomes of over 1,800 accessions of wild and cultivated rice, including 240 aus and 73 aromatic varieties, and analysed the haplotype diversity of the taxonomic groups. We confirm the deep phylogenetic divergence between the main chloroplast haplotypes of japonica and indica, and reveal unique profiles of chloroplast diversity in aus and aromatic rice. Our results indicate that the latter two groups are not simple derivatives of indica and japonica, respectively, but originated from independent and/or reticulate domestication processes. Absence of phylogeographic patterns in the wild distribution of chloroplast haplogroups did not allow firm conclusions about geographic origins and the role of inter-group gene flow. Nonetheless, our results suggest that the domestication of indica, japonica, aus and aromatic rice operated on genetically different gene pools and followed different dynamics.
\end{abstract}

Key words: chloroplast network, bottleneck, whole-genome sequencing data, PCA

\section{Introduction}

Cultivated Asian rice (Oryza sativa L.) is one of the oldest and most important staple crops worldwide. It is well established that $O$. sativa originated from a wild progenitor species Oryza rufipogon Griff., but the number of domestication events and the population genetic details of the process remain controversial (He et al. 2011; Yang et al. 2012; Molina et al. 2011; Huang et al. 2012; Civáň et al. 2015; Huang and Han 2015; Civáň and Brown 2016). Based on ecology, genetics and culinary properties, $O$. sativa can be divided into five groups - japonica (subdivided into tropical and temperate ecological groups), indica, aus, and aromatic rice (Garris et al 2005; Zhao et al. 2011; Civáň et al. 2015). Japonica varieties produce glutinous grain that is particularly sticky after cooking compared to long-grained indica and aus rice, the latter consisting of droughttolerant, early-maturing varieties. Aromatic rice includes cultivars with specific flavors popular in Pakistan and northern India (basmati) and Iran (sadri). Reproductive separation of O. sativa and $O$. rufipogon is incomplete and gene flow between these species has been well-documented due to concerns of transgene escape from genetically modified rice (Song et al. 2003; Chen et al. 2004; Wang et al. 2006; Shivrain et al. 2007). An incomplete reproductive barrier was also described between indica and japonica rice (Harushima et al. 2002; Yang et al. 2012), but data on aus and 
bioRxiv preprint doi: https://doi.org/10.1101/094482; this version posted December 15, 2016 . The copyright holder for this preprint (which was not certified by peer review) is the author/funder, who has granted bioRxiv a license to display the preprint in perpetuity. It is made available under aCC-BY-NC-ND 4.0 International license.

aromatic crossability are missing.

The question of the genetic and geographic origins of the four rice groups (indica, japonica, aus and aromatic) is still debated. In recent years, the dominant view of the origins of indica and japonica has hypothesized that japonica was domesticated first in southern China and indica was derived later in other regions by hybridization of japonica with locally adapted wild rice (Huang et al. 2012). This domestication model is consistent with the notion that gene flow significantly shaped the genomes of indica and japonica (He et al. 2011; Yang et al. 2012), and recent analyses of rice genes apparently targeted by domestication are often interpreted accordingly (Hua et al. 2015; Oikawa et al. 2015; Si et al. 2016). Nonetheless, the hypothesis that some crucial domestication traits of indica have been derived from japonica was challenged by our group (Civáň et al. 2015). It also becomes apparent that the marginal aus crop has a unique domestication history (Zhao et al. 2011; Schatz et al. 2014; Civáň et al. 2015). Further genetic structure within the aus group was recently discovered by Travis et al. (2015) who differentiated two genetic subgroups within 250 aus cultivars, one of which is associated with the term 'boro', used to describe the winter growing season in Bangladesh and Assam. Although the aromatic varieties have been traditionally associated with the japonica group (Garris et al. 2005; Zhao et al. 2011), we suggested that aromatic rice was rather derived from crosses between aus and japonica (Civáň et al. 2015).

Besides the genetic diversity present in the nuclear genetic compartment, cytoplasmic (chloroplast and mitochondrial) genomes provide alternative sources of genealogical data often used for deep phylogenetic reconstructions (chloroplast genomes in plants), as well as phylogeography of populations (e.g. human mitochondrial genome). Cytoplasmic genomes are typically uniparentally inherited and are generally considered to be non-recombining units of genetic material, which means that their genealogical history is analogous to the genealogical history of a single gene. Consequently, the phylogeny reconstructed from chloroplast or mitochondrial genomes can differ from the species- or population-level phylogeny for reasons of lineage sorting, even in the total absence of introgression (Avise and Wollenberg 1997). Because of this, chloroplast genomes may not be reliable for species-tree reconstruction in recently diverged taxa, even when a sufficient number of phylogenetically informative characters are present. However, domestication is a special case of very recent and relatively rapid speciation typically accompanied by a severe bottleneck due to strong artificial selection. In such a case, the chloroplast genome diversity of a crop may be extremely reduced in respect to the diversity of the wild populations, and this reduction can provide indications about the course of domestication. Chloroplast uniformity across the entire crop can be interpreted as evidence of a single origin and a rapid domestication, while high chloroplast diversity indicates multiple origins or a lengthy domestication process with inter-group gene flow. Using this rationale, population-scale chloroplast datasets were utilized to infer single origins of domesticated sunflower (Wills and Burke 2006) and peanut (Grabiele et al. 2012), multiple domestications of lima bean (Andueza-Noh et al. 2013), as well as to make implications about intra and inter-specific hybridizations that contributed to the current genetic diversity of the domesticated species (Delplancke et al. 2011; Nikiforova et al. 2013; Li et al. 2013).

Chloroplast genome data has also been used to study the relationships among the Oryza species (Kim et al. 2015; Wambugu et al. 2015) and particularly the context of rice domestication (Kawakami et al. 2007; Tong et al. 2016; Kumagai et al. 2016). The datasets used either comprise short chloroplast DNA fragments reconstructed for hundreds of rice accessions (Kawakami et al. 2007; Kumagai et al. 2016), or involve the entire chloroplast genomes of dozens (Kim et al. 2015; Wambugu et al. 2015) to hundreds rice accessions (Tong et al. 2016). These studies revealed that indica and japonica rice carry generally distinct chloroplast lineages - each group being more closely related to particular wild haplotypes than to one another - supporting the hypothesis of multiple origins of O. sativa (Kawakami et al. 2007; Tong et al. 2016; Kumagai et al. 2016). However, aus and aromatic rice varieties are not represented in these datasets and the origin and diversity of the cytoplasmic lineages in these two groups remain unknown.

In this study, we utilized available whole-genome sequencing datasets (Huang et al. 2012; 3K RGP 2014) and reconstructed complete or nearly-complete chloroplast genomes of $\sim 1,800$ 
bioRxiv preprint doi: https://doi.org/10.1101/094482; this version posted December 15, 2016 . The copyright holder for this preprint (which was not certified by peer review) is the author/funder, who has granted bioRxiv a license to display the preprint in perpetuity. It is made available under aCC-BY-NC-ND 4.0 International license.

Oryza accessions, including 240 aus and 73 aromatic accessions. We compared the chloroplast DNA diversity to the nuclear genome diversity and discuss the revealed patterns in the context of the domestication process and possible post-domestication gene flow.

\section{Results and Discussion}

\section{Chloroplast haplotypes of Oryza populations and inter-group admixture}

We used previously published Illumina whole-genome sequencing datasets produced by the shotgun approach (Huang et al. 2012; 3K RGP 2014) to perform a population-scale assembly and analysis of complete chloroplast genomes. We report here complete or near-complete chloroplast genomes for 1,823 accessions of wild (460 accessions) and cultivated rice (519 indica, 409 temperate japonica, 75 tropical japonica, 240 aus/boro, 73 aromatic; Table S1) assembled through a highly efficient yet simple bioinformatics pipeline. In the 1,823 assemblies, typically about $7.4 \%$ of the raw data mapped to the chloroplast genome, resulting in each base covered $601 \times$ and $189 \times$ on average for the O. rufipogon and O. sativa samples respectively (with 184.7 and 86.8 mean standard deviation of coverage; Table S1).

A 120,613-nucleotide long alignment of the reconstructed genomes revealed 216 polymorphic positions with $>0.5 \%$ frequency in the entire super-population. Only a single position out of the 216 SNPs had three character states, indicating that the phylogenetic signal is not distorted by multiple substitutions and the dataset is generally free of substitutional saturation. The $\mathrm{Ka} / \mathrm{Ks}$ ratio $(0.24)$ calculated for all coding regions indicates purifying selection, although parts of some genes ( $m a t K, r p o A, r p o C 2$ ) may be evolving under positive selection. A data matrix of the 216 SNPs and 1,763 rice accessions containing $0.7 \%$ missing data was used to construct a median-joining network (Fig. 1). The 216 polymorphic positions define 107 distinct haplotypes that can be assigned into six major haplogroups (Fig. 1). In total, 67 haplotypes were found within wild rice and 55 haplotypes within cultivated rice.

Each cultivated group has a unique profile of chloroplast haplotypes. In temperate and tropical japonica we found 21 haplotypes in total, 20 of these in haplogroup A with three haplotypes being particularly common - A02 (31.4\% of japonica samples), A03 (28.5\%) and A01 (24.3\%) and 1 haplotype in haplogroup B (B01-1\%). Within the indica group, we found 33 haplotypes in total, with haplotype B01 being the most common (60.9\%). While $83.3 \%$ of indica samples fall within haplogroup $B, 15.2 \%$ are found within haplogroup $A$, sharing several haplotypes with japonica (particularly the haplotype A01). The clear differentiation of the main japonica and indica haplogroups (haplogroups $A$ and $B$, respectively) is in agreement with earlier studies that resolved the chloroplast genomes of these two groups in two different $O$. rufipogon/sativa clades (Kim et al. 2015; Wambugu et al. 2015). Despite the deep divergence of the japonica A-lineages and the indica B-lineages, a significant portion of indica accessions carries non-B haplotypes, and this observation is consistent with the findings of Kumagai et al. (2016).

Within the aus group, we found 18 haplotypes, among which B01, B02, A04 and A01 $(34.2 \%, 23.3 \%, 16.3 \%$ and $10.8 \%$, respectively) are the most frequent. Hence, the aus accessions carry the indica-like B haplotypes (B01 and its derivative B02), and the japonica-like A haplotypes (A01), but also haplotypes that are absent in japonica and indica (A04). Interestingly, the haplotype A04 is shared with aromatic rice, where $60.2 \%$ of accessions carry A04 or its derivative A09. Six additional haplotypes were found in the aromatic accessions, with A01, A02, A14 and B01 being relatively frequent (all approximately $10 \%$ ).

\section{Substitution rates for chloroplast genomes are relatively slow and have been} estimated at $1.52 \times 10^{-9}$ (Yamane et al. 2006) and $1.06 \times 10^{-9}$ (Middleton et al. 2014) per site per year in grasses. Within the rice chloroplast network, the largest distance between wild haplotypes is 90 substitutions separating the nodes $\mathrm{A} 03$ and 01 . The latter comprises $\mathrm{O}$. meridionalis and $\mathrm{O}$. rufipogon sampled from Australia and Indonesia, confirming the unique identity of Australian wild rice reported previously (Waters et al. 2011). The most frequent haplotypes of japonica and indica (A01 and B01, respectively) are separated by 40 substitutions, which represents $116,000-166,000$ 
years of divergence when the substitution rates by Yamane et al. (2006) and Middleton et al. (2014) are used. This figure is lower than the indica-japonica divergence estimates obtained from nuclear loci (Vitte et al. 2004; Ma \& Bennetzen 2004; Zhu et al. 2005), implying that either the rate of chloroplast evolution or the nuclear divergence time have been overestimated. On average, cultivated haplotypes are separated by only 0.27 substitutions from their closest wild haplotype, indicating that most of the observed diversity pre-dates domestication.

Among the cultivated groups, aus has the highest diversity, while japonica has relatively high haplotype diversity, but the lowest haplogroup diversity (Table 1). As expected, chloroplast diversity in O. rufipogon is higher than in any of the O. sativa groups, both in terms of haplotype and haplogroup diversity. The O. rufipogon haplogroups do not display a clear phylogeographic pattern, each haplogroup encompassing a large geographic area stretching from India to southeast Asia and China (Fig. 2). This observation is in agreement with Liu et al. (2015) and Kumagai et al. (2016), who found no correlation between genetic groups and geographic regions. Since the divergence of the haplogroups precedes the last glacial maximum and perhaps also the previous interglacial (implied by the number of substitutions and the rate of chloroplast evolution), the absence of any phylogeographic pattern is probably due to repeated extinctions and recolonizations of wild populations during Quaternary glaciation cycles. Unfortunately, the wide distribution of the chloroplast haplogroups in the wild does not allow any inference of the geographic origins of the cultivated groups.

A principal component analysis (PCA) performed on a genome-wide collection of nuclear single nucleotide polymorphisms (SNPs) revealed differences between the nuclear and chloroplast diversity patterns. The four cultivated groups are well differentiated and form clusters associated with three groups of $O$. rufipogon (Fig. 3A). All cultivated groups are differentiated by the first two eigenvectors with statistical significance, except for aus and indica along the first eigenvector. However, when the rice accessions in the same analysis are recoded according to their chloroplast haplogroups, the PCA clusters become heterogenous, indicating that the chloroplast haplogroups poorly represent the diversity of the nucleus. This is probably a reflection of the non-recombining nature of the chloroplast genome that passes through generations as a single locus strongly affected by lineage sorting (Avise and Wollenberg 1997). Discrepancies between the nuclear and cytoplasmic phylogenetic signals were also observed in other crop-wild systems (Nikiforova et al. 2013; Delplancke et al. 2013), which warns against phylogenetic inference solely based on chloroplast data from recently diverged species or populations.

Nonetheless, the network of chloroplast diversity (Fig. 1) reveals one general distinction among the cultivated groups - while practically all japonica accessions have closely related haplotypes (98.8\% derived from a single node A15), the other three groups contain rather unrelated haplotypes in non-negligible proportions. This observation is consistent with previous findings of generally lower levels of genetic diversity in the nuclear genome of japonica compared to indica (Caicedo et al. 2007; He et al. 2011; Huang et al. 2012) and suggests differences in the population dynamics during or after domestication. Two general models can be hypothesized to explain the observed pattern. (i) The chloroplast haplotypes of the cultivated groups were inherited vertically from multiple progenitor populations that differed in their genetic diversity. Under this model, the genetic basis of japonica was extremely narrow - either due to a very small ancestral population or a bottleneck caused by strong artificial selection - while the ancestral gene pools of aus, indica and aromatic rice were genetically wider. Under the second model (ii), the (chloroplast) diversity of indica, aus and aromatic rice was enriched by post-domestication admixture. Postdomestication admixture can, however, operate under two different scenarios - the cultivated groups were either domesticated independently from defined wild populations and subsequently admixed with other cultivated/wild gene pools, or all cultivated groups are derived from a single domestication event followed by very dynamic wild admixture events leading to the formation of distinct cultivated groups. The latter scenario was proposed by Huang et al. (2012) who suggest that japonica rice was the originally domesticated crop from which the other $O$. sativa groups were derived. Interestingly, the japonica-typical haplotype A1 is found in non-negligible frequencies in all cultivated groups, which allows the speculation that japonica played some role in the ancestry of indica, aus and aromatic rice. However, since the A haplogroup (and more specifically the Al 
haplogroup) is widely distributed in wild rice populations (Fig. 2A, Fig. 3), it is equally possible that all cultivated groups obtained A-haplotypes vertically from their wild progenitors. The haplotype profiles observed here can also be interpreted in accordance with the alternative domestication scheme we proposed recently (Civáň et al. 2015), in which the indica, japonica and aus groups were domesticated independently, indica and aus having wider and partially overlapping ancestral gene pools, while aromatic varieties arose from hybridizations between japonica and aus. Independent origins of indica and japonica are documented here by the deep divergence of their prevalent haplotypes, while the close association of aus and aromatic rice is supported by sharing the A04 haplotype that is frequent and almost exclusive in these two cultivated groups. The scenario of three independent origins of $O$. sativa and the hybrid ancestry of the aromatic varieties is also supported by the PCA of nuclear variation, where three clusters of $O$. rufipogon are associated with different cultivated groups and the aromatic varieties are positioned in between the japonica and aus clusters (Fig. 3A).

In summary, we provide a comprehensive picture of chloroplast diversity in $O$. rufipogon and $O$. sativa populations. The possibly strong effect of lineage sorting on the genealogical patterns of chloroplast genomes coupled with the absence of a phylogeographic pattern in wild rice does not permit definite conclusions about geographic origins of the cultivated groups or the exact role of inter-group gene flow. Nonetheless, our results provide further support to the notion that japonica has a more limited genetic basis than the other cultivated groups. Furthermore, the unique profiles of chloroplast haplotypes observed in aus and aromatic rice indicate that these groups are not simple derivatives of indica and japonica, but rather originated from independent and/or reticulate domestication processes.

\section{Materials and Methods}

\section{Reconstruction and analysis of chloroplast genomes}

Raw sequencing data for 1,823 wild and cultivated rice accessions (Table S1) published by Huang et al. (2012) and the 3k RGP (2014) were downloaded from the Sequence Read Archive (http://ncbi.nlm.nih.gov/Traces/sra/) and converted into FASTQ format using the fastq-dump command in sratoolkit 2.3.5. The full FASTQ dataset consisted of 12.0 billion Illumina reads totalling $3.1 \mathrm{~TB}$. For each of the 1,823 accessions, reads significantly matching known rice chloroplast genomes (E-value $<1$ e-5) were extracted using the interleave_pairs and filter_by_blast scripts from the seq_crumbs-0.1.9 package. Adapter contamination and low-quality regions were removed from the matching reads by Trimmomatic- 0.33 (Bolger et al. 2014). These procedures yielded 721 million chloroplast-matching reads, totalling $256 \mathrm{~GB}$, equating to $>10$-fold reduction of the original data. The filtered and trimmed datasets were imported into Geneious 6.1 (Biomatters; http://geneious.com) and individually mapped onto the Nipponbare chloroplast genome (KM088016) used as a reference (5 mapping iterations; maximum of $5 \%$ mismatches and $10 \%$ gaps per read; maximum gap size set to 100 ; index word length 13 ; only paired reads matching within the expected distance were mapped). The reconstructed chloroplast genomes with total quality scores are deposited at DOI: $10.5281 /$ zenodo.61977. The second copy of the inverted repeat region was removed from the reconstructed sequences, which were then aligned using MAFFT v7 (Katoh et al. 2002) implemented in Geneious. Chloroplast genomes that were difficult to align due to a high proportion of missing data were further removed and the entire alignment manually corrected. The Ka/Ks ratio was calculated for a sample of 1,352 accessions with no missing data or ambiguities in their coding sequences using DnaSP (Librado and Rozas 2009). Polymorphic sites with frequencies $<0.005$ in the entire superpopulation were extracted from the alignment, treating all gaps as missing data and ignoring inversions and di-/poly-nucleotide changes. The resulting data matrix consisted of 216 sites and 1,763 rice accessions (461 accessions from wild Oryza species; 507 indica; 407 temperate japonica; 240 aus; 75 tropical japonica; 73 aromatic), with $0.7 \%$ missing data points in total. The data matrix was manually converted from FASTA into RDF format and imported into Network 4 (Bandelt et al. 1999) where a median-joining network was constructed. The accessions with intermediate phenotypes were not included in the network, but their haplogroups were recorded and are listed in Table S1. 
Chloroplast divergence times were calculated using the equation $T=D_{X Y} / 2 I$, where $T$ is the divergence time, $D_{X Y}$ is the proportion of differences observed between the sequences $X$ and $Y$, and $l$ is the rate of nucleotide substitution per site per year (Nei, 1987).

\section{PCA of nuclear variation}

The complete genotype dataset for 1,529 wild and cultivated rice accessions consisting of $\sim 8$ million SNPs from all twelve rice chromosomes published by Huang et al. (2012) was downloaded from the Rice Haplotype Map Project database (http://www.ncgr.ac.cn/RiceHap3). Individuals with $>75 \%$ missing data points were removed from the dataset and the accessions of wild rice were further reduced to contain only 120 genotypes that represent the progenitor gene pools of indica, japonica and aus (Civáň $\mathrm{P}$, unpublished data). Characters showing no variation in this subset of samples were removed, yielding a SNP matrix with 701 individuals and 5,759,208 positions. The PCA was performed with smartpca (Patterson et al. 2006), without outlier exclusion and inferring genetic distance from physical distance.

\section{Acknowledgements}

This work was supported by European Research Council grant 339941 awarded to TAB.

\section{References}

3K RGP. 2014. The 3,000 rice genomes project. GigaScience 3:7.

Andueza-Noh RH, Serrano-Serrano ML, Sánchez MIC, del Pino IS, Camacho-Pérez L, CoelloCoello J, Cortes JM, Debouck DG, Martínez-Castillo J. 2013. Multiple domestications of the Mesoamerican gene pool of lima bean (Phaseolus lunatus L.): evidence from chloroplast DNA sequences. Genet Resour Crop Evol. 60:1069-1086.

Avise JC, Wollenberg K. 1997. Phylogenetics and the origin of species. Proc Natl Acad Sci USA. 94:7748-7755.

Bandelt HJ, Forster P, Röhl A. 1999. Median-joining networks for inferring intraspecific phylogenies. Mol Biol Evol. 16:37-48.

Bolger AM, Lohse M, Usadel B. 2014. Trimmomatic: A flexible trimmer for Illumina Sequence Data. Bioinformatics btu170.

Caicedo AL, Williamson SH, Hernandez RD, Boyko A, Fledel-Alon A, York TL, Polato NR, Olsen KM, Nielsen R, McCouch SR, et al. 2007. Genome-wide patterns of nucleotide polymorphism in domesticated rice. PLoS Genet. 3:e163.

Chen LJ, Lee DS, Song ZP, Suh HS, Lu B-R. 2004. Gene flow from cultivated rice (Oryza sativa) to its weedy and wild relatives. Ann Bot. 93:67-73.

Civáň P, Craig H, Cox CJ, Brown TA. 2015. Three geographically separate domestications of Asian rice. Nature Plants 1:15164.

Delplancke M, Alvarez N, Espíndola A, Joly H, Benoit L, Brouck E, Arrigo N. 2012. Gene flow among wild and domesticated almond species: insights from chloroplast and nuclear DNA markers. Evol Appl. 5:317-329.

Garris AJ, Tai TH, Coburn J, Kresovich S, McCouch S. 2005. Genetic structure and diversity in Oryza sativa L. Genetics 169:1631-1638.

Grabiele M, Chalup L, Robledo G, Seijo G. 2012. Genetic and geographic origin of domesticated peanut as evidenced by $5 \mathrm{~S}$ rDNA and chloroplast DNA sequences. Plant Syst Evol. 298:1151-1165.

Harushima Y, Nakagahra M, Yano M, Sasaki T, Kurata N. 2002. Diverse variation of reproductive 
barriers in three intraspecific rice crosses. Genetics 160:313-322.

He Z, Zhai W, Wen H, Tang T, Wang Y, Lu X, Greenberg AJ, Hudson RR, Wu C-I, Shi S. 2011. Two evolutionary histories in the genome of rice: the roles of domestication genes. PLoS Genet. 7:e1002100.

Hua L, Wang DR, Tan L, Fu Y, Liu F, Xiao L, Zhu Z, Fu Q, Sun X, Gu P, et al. 2015. LABA1, a domestication gene associated with long, barbed awns in wild rice. Plant Cell 27:18751888.

Huang X, Han B. 2015. Rice domestication occurred through single origin and multiple introgressions. Nature Plants 2:15207.

Huang X, Kurata N, Wei X, Wang Z-X, Wang A, Zhao Q, Zhao Y, Liu K, Lu H, Li W, et al. 2012. A map of rice genome variation reveals the origin of cultivated rice. Nature 490:497-503.

Katoh K, Misawa K, Kuma K, Miyata T. 2002. MAFFT: a novel method for rapid multiple sequence alignment based on fast Fourier transform. Nucleic Acids Res. 30:3059-3066.

Kawakami S-i, Ebana K, Nishikawa T, Sato Y-i, Vaughan DA, Kadowaki K-i. 2007. Genetic variation in the chloroplast genome suggests multiple domestications of cultivated Asian rice (Oryza sativa L.). Genome 50:180-187.

Kim K, Lee S-C, Lee J, Yu Y, Yang K, Choi B-S, Koh H-J, Waminal NE, Choi H-I, Kim NH, et al. 2015. Complete chloroplast and ribosomal sequences for 30 accessions elucidate evolution of Oryza AA genome species. Sci Rep. 5:15655.

Kumagai M, Kanehara M, Shoda S, Fujita S, Onuki S, Ueda S, Wang L. 2016. Rice varieties in archaic east Asia: reduction of its diversity from past to present times. Mol Biol Evol. 33:2496-2505.

Librado P, Rozas J. 2009. DnaSP v5: A software for comprehensive analysis of DNA polymorphism data. Bioinformatics 25:1451-1452.

Li L-F, Wang H-Y, Zhang C, Wang X-F, Shi F-X, Chen W-N, Ge X-J. 2015. Origins and domestication of cultivated banana inferred from chloroplast and nuclear genes. PLoS One 8:e80502.

Liu R, Zheng X-M, Zhou L, Zhou H-F, Ge S. 2015. Population genetic structure of Oryza rufipogon and Oryza nivara: implications for the origin of O. nivara. Mol Ecol. 24: 5211-5228.

Ma J, Bennetzen JL. 2004. Rapid recent growth and divergence of rice nuclear genomes. Proc Natl Acad Sci USA. 101:12404-12410.

Middleton CP, Senerchia N, Stein N, Akhunov ED, Keller B, Wicker T, Kilian B. 2014. Sequencing of chloroplast genomes from wheat, barley, rye and their relatives provides a detailed insight into the evolution of the Triticeae tribe. PLoS One 9:e85761.

Molina J, Sikora M, Garud N, Flowers JM, Rubinstein S, Reynolds A, Huang P, Jackson S, Schaal BA, Bustamante CD, et al. 2011. Molecular evidence for a single evolutionary origin of domesticated rice. Proc Natl Acad Sci USA. 108:8351-8356.

Nei M. 1987. Molecular Evolutionary Genetics. New York: Columbia University Press.

Nikiforova SV, Cavalieri D, Velasco R, Goremykin V. Phylogenetic analysis of 47 chloroplast genomes clarifies the contribution of wild species to the domesticated apple maternal line. Mol Biol Evol. 30:1751-1760.

Patterson N, Price AL, Reich D. 2006. Population structure and eigenanalysis. PLoS Genet. 2:e190.

Schatz MC, Maron LG, Stein JC, Hernandez Wences A, Gurtowski J, Biggers E, Lee H, Kramer M Antoniou E, Ghiban E, et al. 2014. Whole genome de novo assemblies of three divergent strains of rice, Oryza sativa, document novel gene space of aus and indica. Genome Biol. 15:506. 
Shivrain VK, Burgos NR, Anders MM, Rajguru SN, Moore J, Sales MA. 2007. Gene flow between Clearfield ${ }^{\mathrm{TM}}$ rice and red rice. Crop Prot. 26:349-356.

Si L, Chen J, Huang X, Gong H, Luo J, Hou Q, Zhou T, Lu T, Zhu J, Shangguan Y, et al. 2016. OsSPL13 controls grain size in cultivated rice. Nature Genet. 48:447-457.

Song ZP, Lu B-R, Zhu YG, Chen JK. 2003. Gene flow from cultivated rice to the wild species Oryza rufipogon under experimental field conditions. New Phytol. 157:657-665.

Tong W, Kim T-S, Park Y-J. 2016. Rice chloroplast genome variation architecture and phylogenetic dissection in diverse Oryza species assessed by whole-genome resequencing. Rice 9:57.

Vitte C, Ishii T, Lamy F, Brar D, Panaud O. 2004. Genomic paleontology provides evidence for two distinct origins of Asian rice (Oryza sativa L.). Mol Gen Genomics 272:504-511.

Wambugu PW, Brozynska M, Furtado A, Waters DL, Henry RJ. 2015. Relationships of wild and domesticated rices (Oryza AA genome species) based upon whole chloroplast genome sequences. Sci Rep. 5:13957.

Wang F, Yuan Q-H, Shi L, Qian Q, Liu W-G, Kuang B-G, Zeng D-L, Liao Y-L, Cao B, Jia S-R. 2006. A large-scale field study of transgene flow from cultivated rice (Oryza sativa) to common wild rice (O. rufipogon) and barnyard grass (Echinochloa crusgalli). Plant Biotechnol J. 4:667-676.

Waters DLE, Nock CJ, Ishikawa R, Rice N, Henry RJ. 2011. Chloroplast genome sequence confirms distinctness of Australian and Asian wild rice. Ecol Evol. 2:211-217.

Wills DM, Burke JM. Chloroplast DNA variation confirms a single origin of domesticated sunflower (Helianthus annuus L.). J Hered. 97:403-408.

Yamane K, Yano K, Kawahara T. 2006. Pattern and rate of indel evolution inferred from whole chloroplast intergenic regions in sugarcane, maize and rice. DNA Res. 13:197-204.

Yang C-c, Kawahara Y, Mizuno H, Wu J, Matsumoto T, Itoh T. 2012. Independent domestication of Asian rice followed by gene flow from japonica to indica. Mol Biol Evol. 29:1471-1479.

Yang J, Zhao X, Cheng K, Du H, Ouyang Y, Chen J, Qiu S, Huang J, Jiang Y, Jiang L, et al. 2012. A killer-protector system regulates both hybrid sterility and segregation distortion in rice. Science 337:1336-1340.

Zhao K, Tung C-W, Eizenga GC, Wright MH, Ali ML, Price AH, Norton GJ, Islam MR, Reynolds A, Mezey J, et al. 2011. Genome-wide association mapping reveals a rich genetic architecture of complex traits in Oryza sativa. Nature Commun. 2:467.

Zhu Q, Ge S. 2005. Phylogenetic relationships among A-genome species of the genus Oryza revealed by intron sequences of four nuclear genes. New Phytol. 167:249-265. 
bioRxiv preprint doi: https://doi.org/10.1101/094482; this version posted December 15, 2016. The copyright holder for this preprint (which was not certified by peer review) is the author/funder, who has granted bioRxiv a license to display the preprint in perpetuity. It is made available under aCC-BY-NC-ND 4.0 International license.

\section{Tables}

Table 1. Chloroplast diversity in wild and cultivated rice.

\begin{tabular}{lll}
\hline & Haplotype diversity $^{\mathbf{a}}$ & Haplogroup diversity $^{\mathbf{b}}$ \\
\hline O. rufipogon & 0.9587 & 0.7450 \\
indica & 0.6152 & 0.2836 \\
aus & 0.7903 & 0.5133 \\
japonica & 0.7591 & 0.0103 \\
aromatic & 0.7561 & 0.2432 \\
\hline
\end{tabular}

a calculated as $H=\frac{N}{N-1}\left(1-\sum_{i} x_{i}^{2}\right)$ where $x_{i}$ is the relative haplotype frequency of each haplotype in the given population and $N$ is the sample size

${ }^{\mathrm{b}}$ calculated as above, except using haplogroups instead of haplotypes

\section{Figure legends}

Fig. 1. Median-joining network of rice chloroplast haplotypes. This is an unrooted network, however the root is most likely placed among the "o" (outgroup) haplotypes. This branch includes African O. barthii and O. glaberrima (04), O. longistaminata (07), O. meridionalis (01-03, o6) as well as $O$. rufipogon accessions sampled in Australia and Indonesia. Due to the uncertain root placement, the "o" haplotypes are not assigned to a formal haplogroup. (Upper panel) Six haplogroups designated $\mathrm{A}-\mathrm{F}$. Edge lengths are proportional to the number of polymorphisms separating the nodes and node sizes are proportional to the number of samples. As the group composition of haplogroup A is clearly heterogenous, we subdivided it further into haplogroup Al (typical for japonica accessions) and All (japonica accessions absent). (Lower panel) Six haplogroups $(\mathrm{A}-\mathrm{F})$ and 107 haplotypes are labelled. Each node represents a distinct haplotype identified by the haplogroup letter and the node number. The haplotypes are numbered according to their ordered frequency, with the haplotype .1 being the most frequent. Edge lengths are proportional to the number of polymorphisms separating the nodes, except the edge between the haplotypes 03 and o7 (shortened).

Fig. 2. Geographic distribution of $O$. rufipogon chloroplast haplogroups. (A-F) Haplogroups A-F. A single map location may represent multiple samples. One Indian and 17 Chinese accessions are not shown due to their geographic data being unavailable. Maps prepared in Google Earth v7.1.5.1557.

Fig. 3. PCA of the cultivated groups and the identified ancestral gene pools. A total of $5,759,208$ genome-wide nuclear SNPs were transformed into 700 axes of variation. The first two axes represent $9.7 \%$ of the total variation. (A) The first two eigenvectors with rice accessions coloured according to their domestication class. (B) The first two eigenvectors with rice accessions coloured according to their chloroplast haplogroups. It is apparent that rice samples with unrelated chloroplast haplogroups can be very similar at the nuclear level (note the dense clustering of indica samples carrying different haplotypes). 
bioRxiv preprint doi: https://doi.org/10.1101/094482; this version posted December 15, 2016. The copyright holder for this preprint (which was not certified by peer review) is the author/funder, who has granted bioRxiv a license to display the preprint in perpetuity. It is made available under aCC-BY-NC-ND 4.0 International license.

\section{Figures}

Fig. 1

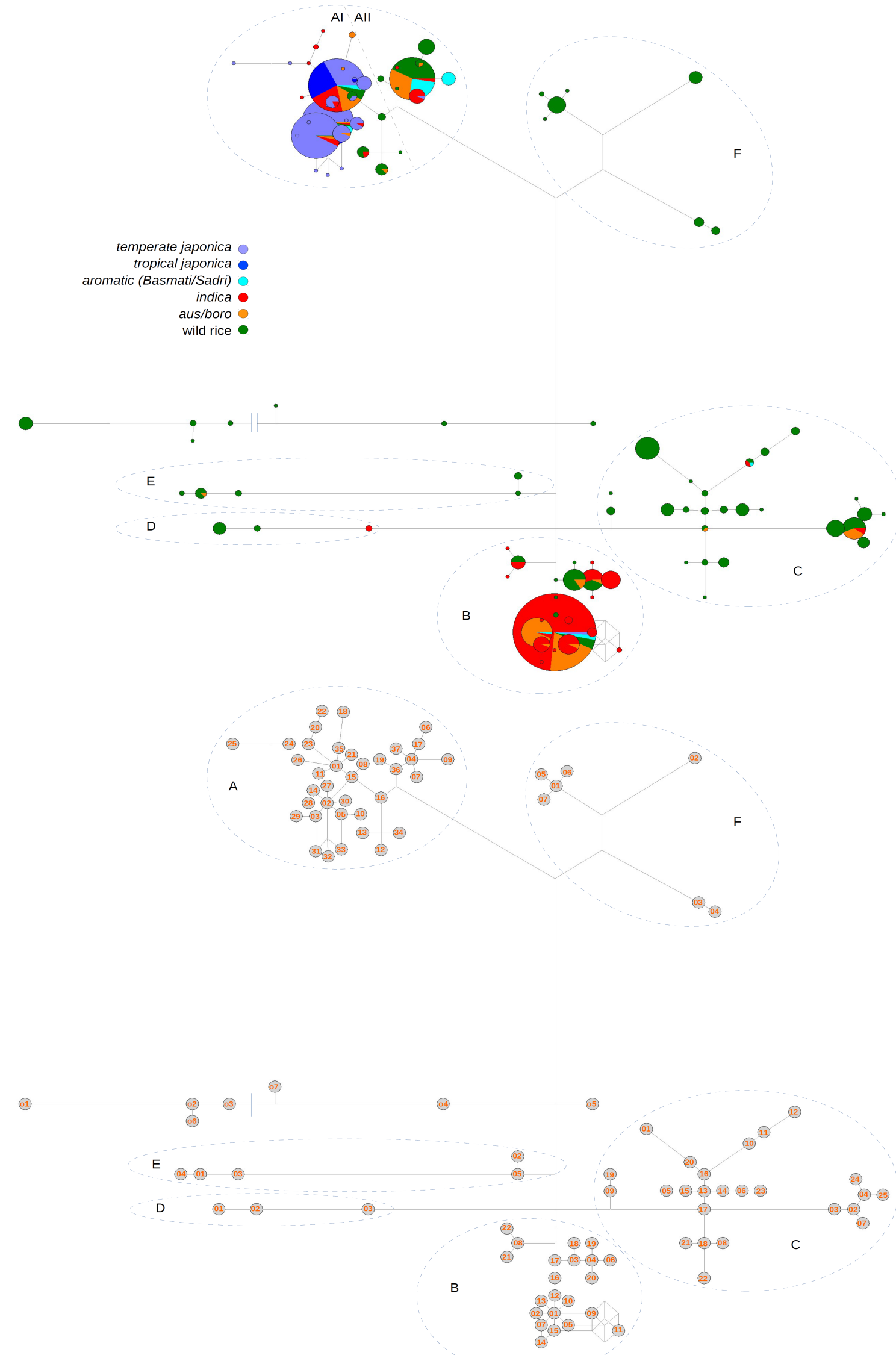


bioRxiv preprint doi: https://doi.org/10.1101/094482; this version posted December 15, 2016. The copyright holder for this preprint (which was not certified by peer review) is the author/funder, who has granted bioRxiv a license to display the preprint in perpetuity. It is made available under aCC-BY-NC-ND 4.0 International license.

Fig. 2

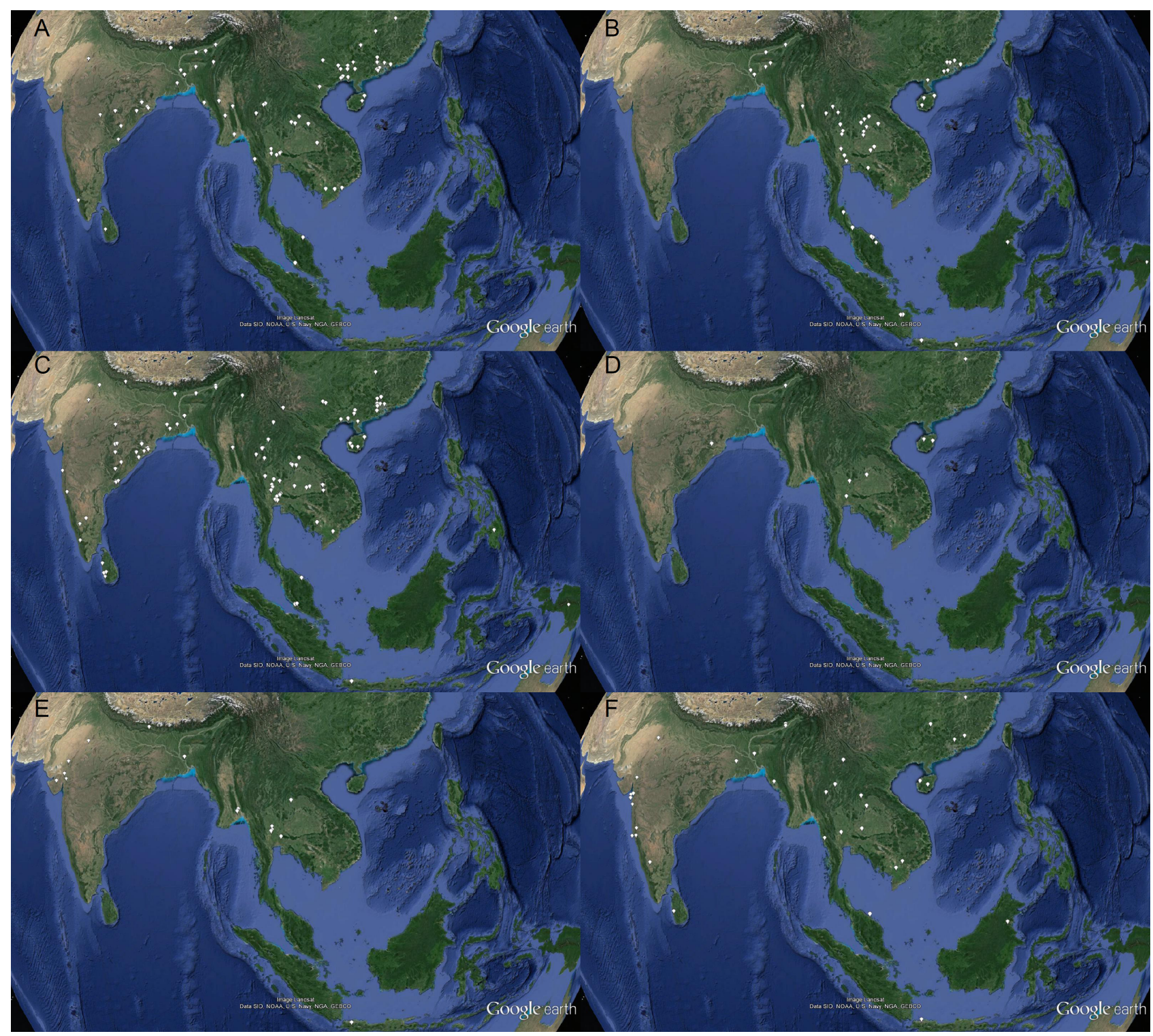


bioRxiv preprint doi: https://doi.org/10.1101/094482; this version posted December 15, 2016. The copyright holder for this preprint (which was not certified by peer review) is the author/funder, who has granted bioRxiv a license to display the preprint in perpetuity. It is made available under aCC-BY-NC-ND 4.0 International license.

Fig. 3
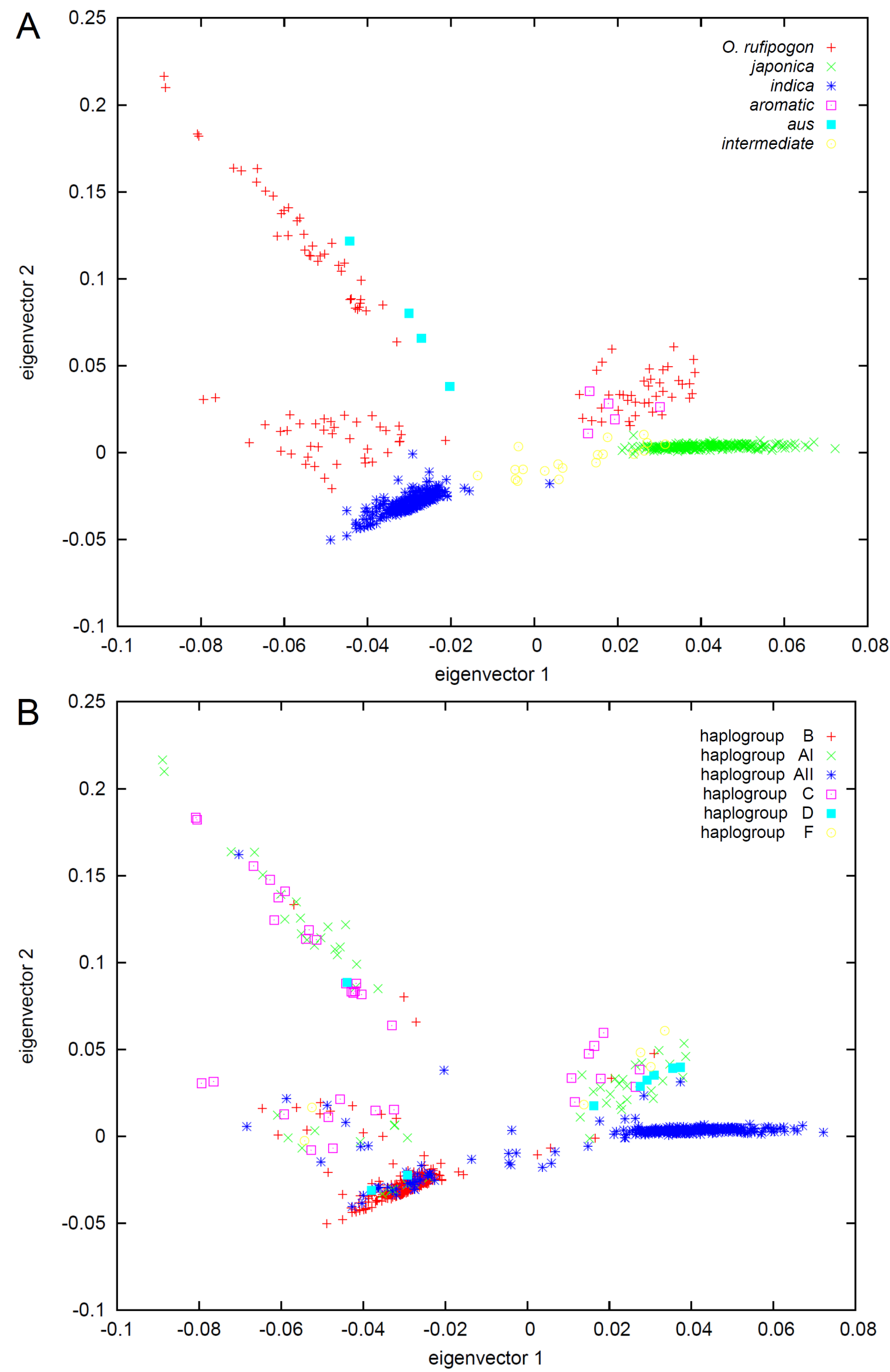